\title{
ON A QUESTION OF GROSS CONCERNING UNIQUENESS OF ENTIRE FUNCTIONS
}

\author{
HONG-XUN YI
}

In this paper, we prove that there exist two finite sets $S_{1}$ (with 1 element) and $S_{2}$ (with 3 elements) such that any two entire functions $f$ and $g$ satisfying $E_{f}\left(S_{j}\right)=$ $E_{g}\left(S_{j}\right)$ for $j=1,2$ must be identical. This answers a question posed by Gross. Examples are provided to show that this result is sharp.

\section{INTRODUCTION}

Let $h$ be a nonconstant entire function, and let $S$ be a subset of distinct elements in $\mathbb{C}$. Define (see [10])

$$
\begin{aligned}
& E_{h}(S)=\bigcup_{a \in S}\{z \mid h(z)=a, \text { counting multiplicities }\} \\
& \vec{E}_{h}(S)=\bigcup_{a \in S}\{z \mid h(z)=a, \text { ignoring multiplicities }\}
\end{aligned}
$$

Let $f$ and $g$ be two nonconstant entire functions, and let $S$ be a subset of distinct elements in $\mathbb{C}$. If $E_{f}(S)=E_{g}(S)$, we say $f$ and $g$ share the set $S$ CM (counting multiplicity). If $\bar{E}_{f}(S)=\bar{E}_{g}(S)$, we say $f$ and $g$ share the set $S$ IM (ignoring multiplicity). As a special case, let $S=\{a\}$, where $a \in \mathbb{C}$. If $E_{f}(\{a\})=E_{g}(\{a\})$, we say $f$ and $g$ share the value $a$ CM. If $\bar{E}_{f}(\{a\})=\bar{E}_{g}(\{a\})$, we say $f$ and $g$ share the value $a \mathrm{IM}$ (see [2]).

In 1976, Gross asked the following question:

Question 1. (See [1, Question 6].) Can one find two finite sets $S_{j} \quad(j=1,2)$ such that any two entire functions $f$ and $g$ satisfying $E_{f}\left(S_{j}\right)=E_{g}\left(S_{j}\right)$ for $j=1,2$ must be identical?

In 1994, the present author [6] proved the following theorem, which answered the above Question 1 in the affirmative.

\section{Received 20th August, 1997}

Project supported by the National Natural Science Foundation of China.

Copyright Clearance Centre, Inc. Serial-fee code: 0004-9729/98 \$A2.00+0.00. 
Theorem A. Let $S_{1}=\left\{\omega \mid \omega^{n}-1=0\right\}$ and $S_{2}=\{a\}$, where $n \geqslant 5, a \neq 0$ and $a^{2 n} \neq 1$. If $f$ and $g$ are entire functions such that $E_{f}\left(S_{j}\right)=E_{g}\left(S_{j}\right)$ for $j=1,2$, then $f \equiv g$.

In [1] Gross wrote: "If the answer to Question 6 is affirmative, it would be interesting to know how large both sets would have to be."

Now it is natural to ask the following question:

QuEsTION 2. What are the smallest cardinalities of $S_{1}$ and $S_{2}$ respectively, where $S_{1}$ and $S_{2}$ are two finite sets such that any two entire functions $f$ and $g$ satisfying $E_{f}\left(S_{j}\right)=E_{g}\left(S_{j}\right)$ for $j=1,2$ must be identical?

In this paper, we prove the following theorems, which answer Question 2.

TheOREM 1. Let $S_{1}=\{0\}$ and $S_{2}=\left\{\omega \mid \omega^{2}(\omega+a)-b=0\right\}$, where $a$ and $b$ are two nonzero constants such that the algebraic equation $\omega^{2}(\omega+a)-b=0$ has no multiple roots. If $f$ and $g$ are two entire functions satisfying $E_{f}\left(S_{j}\right)=E_{g}\left(S_{j}\right)$ for $j=1,2$, then $f \equiv g$.

REMARK. Let $S_{1}=\{0\}$ and $S_{2}=\{2,-3,-6\}$. It is easy to see that $S_{2}=\{\omega \mid$ $\left.\omega^{2}(\omega+7)-36=0\right\}$. From Theorem 1 we immediately obtain that if $f$ and $g$ are entire functions satisfying $E_{f}\left(S_{j}\right)=E_{g}\left(S_{j}\right)$ for $j=1,2$, then $f \equiv g$.

THEOREM 2. If $S_{1}$ and $S_{2}$ are two finite sets such that any two entire functions $f$ and $g$ satisfying $E_{f}\left(S_{j}\right)=E_{g}\left(S_{j}\right)$ for $j=1,2$ must be identical, then $\max \left\{\#\left(S_{1}\right), \#\left(S_{2}\right)\right\} \geqslant 3$, where $\#(S)$ denotes the cardinality of the set $S$.

Remark. From Theorem 2 we immediately obtain that the smallest cardinalities of $S_{1}$ and $S_{2}$ are 1 and 3 respectively, where $S_{1}$ and $S_{2}$ are two finite sets such that any two entire functions $f$ and $g$ satisfying $E_{f}\left(S_{j}\right)=E_{g}\left(S_{j}\right)$ for $j=1,2$ must be identical. This shows that Theorem 1 is sharp.

\section{Some LemMas}

In this paper, we use the usual notations of Nevanlinna theory of meromorphic functions as explained in [3].

LEMMA 1. (See [9, Lemma 5].) Let $f$ and $g$ be two nonconstant meromorphic functions, and let $c_{1}, c_{2}$ and $c_{3}$ be three nonzero constants. If

$$
c_{1} f+c_{2} g=c_{3}
$$

then

$$
T(r, f)<\bar{N}\left(r, \frac{1}{f}\right)+\bar{N}\left(r, \frac{1}{g}\right)+\bar{N}(r, f)+S(r, f)
$$


Let $h$ be a nonconstant meromorphic function. We denote by $N_{2}(r, h)$ the counting function of poles of $h$, where a simple pole is counted once and a multiple pole is counted two times (see [7]).

Lemma 2. (See [7, Theorem 1].) Let $F$ and $G$ be two nonconstant meromorphic functions such that $F$ and $G$ share the value $1 C M$. If

$$
\limsup _{\substack{r \rightarrow \infty \\ r \in I}} \frac{N_{2}(r, 1 / F)+N_{2}(r, F)+N_{2}(r, 1 / G)+N_{2}(r, G)}{T(r)}<1,
$$

where $T(r)=\max \{T(r, F), T(r, G)\}, I$ denotes any set of infinite linear measure of $0<r<\infty$, then $F \equiv G$ or $F \cdot G \equiv 1$.

LEMMA 3. Let

$$
F=\frac{f^{2}(f+a)}{b}, \quad G=\frac{g^{2}(g+a)}{b},
$$

where $f$ and $g$ are two nonconstant entire functions, $a$ and $b$ are two nonzero constants. Then $F \cdot G \not \equiv 1$.

Proof: If $F \cdot G \equiv 1$, from (2.2) we have

$$
f^{2}(f+a) g^{2}(g+a) \equiv b^{2} .
$$

From this we know that $\mathbf{0}$ and $-a$ are Picard exceptional values of $f$, which is impossible. Thus $F \cdot G \not \equiv 1$.

LEMMA 4. Let $f$ and $g$ be two nonconstant entire functions which share the value 0 IM. If $F \equiv G$, where $F$ and $G$ are given by (2.2), then $f \equiv g$.

Proof: Since $F \equiv G$, we have from (2.2)

$$
f^{2}(f+a)=g^{2}(g+a) \text {. }
$$

Noting $f$ and $g$ share the value $0 \mathrm{IM}$, from (2.3) we know that $f$ and $g$ share $0 \mathrm{CM}$. From (2.3) we have

$$
f^{3}-g^{3}=-a\left(f^{2}-g^{2}\right) .
$$

If $f^{3} \not \equiv g^{3}$, from (2.4) we obtain

$$
g=-\frac{a(h+1)}{(h-u)\left(h-u^{2}\right)},
$$

where $h=f / g$ and $u=\exp ((2 \pi i) / 3)$. From (2.5) we know that $h$ is a nonconstant meromorphic function. Noting $f$ and $g$ share the value $0 \mathrm{CM}$, from $h=f / g$ we know that 0 and $\infty$ are Picard exceptional values of $h$. Since $g$ is a nonconstant entire function, from (2.5) we know that $u$ and $u^{2}$ are Picard exceptional values of $h$, which is impossible. Thus $f^{3} \equiv g^{3}$ and $f^{2} \equiv g^{2}$. From this, we get $f \equiv g$. 


\section{Proof of Theorem 1}

Let $F$ and $G$ be given by (2.2). Thus,

$$
T(r, F)=3 T(r, f)+S(r, f), \quad T(r, G)=3 T(r, g)+S(r, g) .
$$

Set

$$
H=\frac{F^{\prime}}{F-1}-\frac{G^{\prime}}{G-1}
$$

We discuss the following two cases.

Case 1. Suppose that $H \equiv 0$. By integration we have from (3.2)

$$
F-1 \equiv A(G-1)
$$

where $A$ is a nonzero constant. We discuss the following two subcases.

CASE 1.1. Assume that $A=1$. From (3.3) we have $F \equiv G$. By Lemma 4 we get $f \equiv g$.

CAse 1.2. Assume that $A \neq 1$. Suppose 0 is not a Picard exceptional value of $f$ and $g$. Since $f$ and $g$ share the value $0 \mathrm{CM}$, then there exists $z_{0}$ such that $f\left(z_{0}\right)=g\left(z_{0}\right)=0$. From (2.2) we obtain $F\left(z_{0}\right)=G\left(z_{0}\right)=0$. From this and (3.3) we get $A=1$, which is a contradiction. Thus, 0 is a Picard exceptional value of $f$ and $g$. From (3.3) we have

$$
F-A G=1-A
$$

From this we have

$$
T(r, G)=T(r, F)+O(1)
$$

By Lemma 1, we obtain from (2.2), (3.1), (3.4) and (3.5)

$$
\begin{aligned}
3 T(r, f) & \leqslant \bar{N}\left(r, \frac{1}{f+a}\right)+\bar{N}\left(r, \frac{1}{g+a}\right)+S(r, f) \\
& \leqslant 2 T(r, f)+S(r, f)
\end{aligned}
$$

which is impossible.

CAse 2. Suppose that $H \not \equiv 0$. Then $F \not \equiv G$. By $E_{f}\left(S_{2}\right)=E_{g}\left(S_{2}\right)$, we know that $F$ and $G$ share the value $1 \mathrm{CM}$. From (3.2) we have

$$
T(r, H)=m(r, H)+N(r, H)=S(r, F)+S(r, G)
$$


Since $f$ and $g$ share the value $0 \mathrm{CM}, f$ and $g$ have the same zeros. Let $z_{0}$ be a zero of $f$ and $g$. From (2.2) and (3.2) we know that $z_{0}$ is a zero of $H$. From this and (3.6) we get

$$
\bar{N}\left(r, \frac{1}{f}\right)=\bar{N}\left(r, \frac{1}{g}\right) \leqslant N\left(r, \frac{1}{H}\right) \leqslant S(r, F)+S(r, G) .
$$

From this, (2.2) and (3.1) we obtain

$$
\begin{aligned}
N_{2}\left(r, \frac{1}{F}\right)+N_{2}\left(r, \frac{1}{G}\right) & \leqslant N\left(r, \frac{1}{f+a}\right)+N\left(r, \frac{1}{g+a}\right)+S(r, F)+S(r, G) \\
& \leqslant \frac{1}{3} T(r, F)+\frac{1}{3} T(r, G)+S(r, F)+S(r, G) .
\end{aligned}
$$

Thus,

$$
\limsup _{\substack{r \rightarrow \infty \\ r \in I}} \frac{N_{2}\left(r, \frac{1}{F}\right)+N_{2}(r, F)+N_{2}\left(r, \frac{1}{G}\right)+N_{2}(r, G)}{T(r)} \leqslant \frac{2}{3}<1,
$$

where $T(r)=\max \{T(r, F), T(r, G)\}, I$ denotes any set of infinite linear measure of $0<r<\infty$. By Lemma 2, we obtain $F \cdot G \equiv 1$. Again by Lemma 3, we get a contradiction.

This completes the proof of Theorem 1 .

\section{Proof of Theorem 2}

\subsection{Some EXAmples.}

EXAMPLE 1. Let $S_{1}=\{a\}$ and $S_{2}=\{b\}$, where $a$ and $b$ are any two finite distinct complex numbers. Let

$$
f(z)=a+(b-a) e^{h(z)}, \quad g(z)=a+(b-a) e^{-h(z)},
$$

where $h(z)$ is a nonconstant entire function. It is easy to show that $E_{f}\left(S_{j}\right)=E_{g}\left(S_{j}\right)$ $(j=1,2)$, but $f \not \equiv g$.

EXAMPle 2. (See [8].) Let $S_{1}=\{a\}$ and $S_{2}=\left\{b_{1}, b_{2}\right\}$, where $a, b_{1}$ and $b_{2}$ are any three finite distinct complex numbers. Let

$$
f(z)=a+\left(b_{1}-a\right) e^{h(z)}, \quad g(z)=a+\left(b_{2}-a\right) e^{-h(z)},
$$

where $h(z)$ is a nonconstant entire function. It is easy to show that $E_{f}\left(S_{j}\right)=E_{g}\left(S_{j}\right)$ $(j=1,2)$, but $f \not \equiv g$. 
EXAmple 3. (See [4].) Let $S_{1}=\left\{a_{1}, a_{2}\right\}$ and $S_{2}=\left\{b_{1}, b_{2}\right\}$, where $a_{1}, a_{2}, b_{1}$ and $b_{2}$ are any four finite distinct complex numbers satisfying $a_{1}+a_{2} \neq b_{1}+b_{2}$. Let

$$
f(z)=d+\left(d-a_{1}\right) e^{h(z)}, \quad g(z)=d+\left(d-a_{2}\right) e^{-h(z)},
$$

where $h(z)$ is a nonconstant entire function, $d=\left(a_{1} a_{2}-b_{1} b_{2}\right) /\left(a_{1}+a_{2}-b_{1}-b_{2}\right)$. It is easy to show that $E_{f}\left(S_{j}\right)=E_{g}\left(S_{j}\right)(j=1,2)$, but $f \not \equiv g$.

Example 4. (See [5].) Let $S_{1}=\left\{a_{1}, a_{2}\right\}$ and $S_{2}=\left\{b_{1}, b_{2}\right\}$, where $a_{1}, a_{2}, b_{1}$ and $b_{2}$ are any four finite distinct complex numbers satisfying $a_{1}+a_{2}=b_{1}+b_{2}$. Let $f(z)$ be a nonconstant entire function, $g(z)=a_{1}+a_{2}-f(z)$. It is easy to show that $E_{f}\left(S_{j}\right)=E_{g}\left(S_{j}\right)(j=1,2)$, but $f \not \equiv g$.

\subsection{Proof of Theorem 2.}

Suppose that $\max \left\{\#\left(S_{1}\right), \#\left(S_{2}\right)\right\}<3$. We proceed to get a contradiction. If $\#\left(S_{1}\right)=\#\left(S_{2}\right)=1$, from Example 1 we have a contradiction. If $\#\left(S_{1}\right)=1$ and $\#\left(S_{2}\right)=2$ or $\#\left(S_{1}\right)=2$ and $\#\left(S_{2}\right)=1$, from Example 2 we have again a contradiction. If $\#\left(S_{1}\right)=\#\left(S_{2}\right)=2$, from Example 3 and Example 4 we can get a contradiction. This completes the proof of Theorem 2.

\section{Concluding Remark}

In fact, in Section 3 of this paper we proved the following theorem, which is an improvement of Theorem 1.

THeOREM 3. Let $S_{1}=\{0\}$ and $S_{2}=\left\{\omega \mid \omega^{2}(\omega+a)-b=0\right\}$, where $a$ and $b$ are two nonzero constants such that the algebraic equation $\omega^{2}(\omega+a)-b=0$ has no multiple roots. If $f$ and $g$ are two entire functions satisfying $\bar{E}_{f}\left(S_{1}\right)=\bar{E}_{g}\left(S_{1}\right)$ and $E_{f}\left(S_{2}\right)=E_{g}\left(S_{2}\right)$, then $f \equiv g$.

Proceeding as in the proof of Theorem 1, we can prove the following result, which is an extension of Theorem 3 .

THEOREM 4. Let $S_{1}=\{0\}$ and $S_{2}=\left\{\omega \mid \omega^{n}(\omega+a)-b=0\right\}$, where $n(\geqslant 2)$ is an integer, $a$ and $b$ are two nonzero constants such that the algebraic equation $\omega^{n}(\omega+\dot{a})-b=0$ has no multiple roots. If $f$ and $g$ are two entire functions satisfying $\bar{E}_{f}\left(S_{1}\right)=\bar{E}_{g}\left(S_{1}\right)$ and $E_{f}\left(S_{2}\right)=E_{g}\left(S_{2}\right)$, then $f \equiv g$.

Let $n(\geqslant 2)$ be an integer, and let $a$ and $b$ be two nonzero constants. It is easy to show that if $b \neq\left(n^{n} a^{n+1}\right) /(n+1)^{n+1}$, the algebraic equation $\omega^{n}(\omega+a)-b=0$ has no multiple roots. Specially, if $b \neq 4 a^{3} / 27$, the algebraic equation $\omega^{2}(\omega+a)-b=0$ has no multiple roots. 


\section{REFERENCES}

[1] F. Gross, 'Factorization of meromorphic functions and some open problems', in Complex Analysis (Proc. Conf. Univ. Kentucky, Lexington, KY, 1976), Lecture Notes in Math. 599 (Springer-Verlag, Berlin, Heidelberg, New York, 1977), pp. 51-69.

[2] G.G. Gundersen, 'Meromorphic functions that share three or four values', J. London Math. Soc. 20 (1979), 457-466.

[3] W.K. Hayman, Meromorphic functions (Clarendon Press, Oxford, 1964).

[4] P. Li, Uniqueness and value sharing of meromorphic functions (Thesis, The Hong Kong University of Science and Technology, 1996).

[5] Hong-Xun Yi, 'On a result of Gross and Yang', Tôhoku Math. J. 42 (1990), 419-428.

[6] Hong-Xun Yi, 'Uniqueness of meromorphic functions and question of Gross', Science in China (Series A) 37 (1994), 802-813.

[7] Hong-Xun Yi, 'Meromorphic functions that share one or two values', Complex Variables Theory Appl. 28 (1995), 1-11.

[8] Hong-Xun $\mathrm{Yi}$, 'The unique range sets for entire or meromorphic functions', Complex Variables Theory Appl. 28 (1995), 13-21.

[9] Hong-Xun Yi and C.C. Yang, 'A uniqueness theorem for meromorphic functions whose n-th derivatives share the same 1-points', J. D'Analyse Math. 62 (1994), 261-270.

[10] Hong-Xun Yi and C.C. Yang, Uniqueness theory of meromorphic functions (Science Press, Beijing, 1995).

Department of Mathematics

Shandong University

Jinan, Shandong 250100

People's Republic of China

e-mail: hxyi@sdu.edu.cn 